\title{
Sudden infant death syndrome in south east Scotland
}

\author{
S E M BARTHOLOMEW, B A MACARTHUR, AND A D BAIN \\ Department of Pathology, Royal Hospital for Sick Children, Edinburgh
}

\begin{abstract}
SUMmaRY Three hundred and fifty eight infants from south east Scotland who died suddenly were classified into four groups. Categories for these groups ranged from where a definite cause of death had been recorded to where no explanation had been provided and no associated disorder was discovered (SIDS). Our results supported the view that there are few differences in the history of cases certified as SIDS and other cases reported as dying suddenly but with an explanation. Groups that most closely matched the SIDS definition employed were reported to be healthier throughout life and freer from illness in the 48 hours before death. From the findings of this study the 'true' SIDS group did not appear as an 'at risk' population. The study group as a whole was not marked by social deprivation, poor mothering, or less privileged families. The importance of intensive investigation, including postmortem examination was emphasised, as misdiagnosis may give a 'falsely' inflated picture of the incidence of the syndrome and could cause unnecessary anxiety.
\end{abstract}

A definition commonly employed in the identification of instances of the sudden infant death syndrome (SIDS) is that proposed in Seattle in 1969:

"The sudden death of any infant or young child which is unexpected by history, and in which a thorough post-mortem examination fails to reveal an adequate cause of death."

In this study data from a group of infants who died suddenly and unexpectedly and who presented as cases of SIDS were analysed to determine to what extent they matched the above definition and to study any differences detected.

The criterion for the diagnosis of SIDS was necessarily pathological as each infant's death was unexpected by history. To carry out this investigation and enable discrimination between the pathological findings, four categories of classification were employed. These classifications, A, AB, B, and $C$, enabled analysis of the various findings and study of the extent to which each may have contributed to the death.

The variables used as parameters for comparison between the groups were selected on the basis of their citation in reviews that dealt with SIDS where possible risk factors, such as preterm delivery, mother's age less than 20 years, and poor social conditions surrounding the family, have been indicated. ${ }^{2} 3$

\section{Procedure}

From 1977 to 1985 , 358 infants aged between 1 week and 2 years who died suddenly and unexpectedly in south east Scotland were referred by the procurator fiscal for postmortem examination. These infants, all of whom presented as cases of SIDS, represented $80 \%$ of fiscal referrals. The $20 \%$, that were excluded failed to meet fully the definition of SIDS given above.

Each case underwent radiological, bacteriological, and virological studies. Biochemical estimates of the sodium, potassium, and urea concentrations in the vitreous humour of the eye were obtained and a detailed postmortem examination performed by a paediatric pathologist. Data on each case were abstracted from obstetric and paediatric records about the pregnancy and birth and any further admissions for the infant. An extensive protocol was used that covered the mothers' antenatal history, parity, interval to last live birth, and regularity of clinic attendances. Problems during pregnancy, such as hypertension, urinary infection, anaemia, and admissions to hospital, were noted.

For the infant, data about mode of birth, gestational age, birth weight, and admissions to the special care baby unit were recorded. Subsequent admissions to hospital were noted by cause, duration of stay, and interval from discharge to death. 
Each family doctor was sent a proforma for completion, which dealt with the mother's previous medical and social history and provided for details regarding any problems with the infant and the family. The health visitors also received a form on which to record their knowledge of the family and infant history, including attendance at the health clinic and visits to the home.

A letter was written to each family with the offer of a visit to the home. This visit was undertaken in every case by the same experienced interviewer two to three weeks after the death when a full history was taken about the infant's health, feeding patterns, circumstances surrounding the death, and household and social organisation of the family. An estimate was made of the reliability and facility of the parents' recall. In 27 cases (7\%) a visit was not carried out as the families had either left the area or intimated their wish not to be interviewed.

Classification into one of four groups was carried out after the interviews and investigations had been completed to reduce the possibility of bias on the part of the investigators.

Group A (26 cases) comprised those cases where a definite cause of death was ascertained. Group AB (53 cases) comprised those cases where an associated disorder was detected that may have been a factor in the death. Group B (193 cases) comprised those cases where an associated disorder was present but was not thought to be an adequate cause of death. Group C (86 cases) comprised those cases where no observable associated disorder was present.

Analysis of the data relating to each variable was carried out to determine whether or not significant differences existed between the groups. Statistical procedures were based on Freund and Scheffé. ${ }^{4}$

The classification of cases into group A was made where an adequate cause of death was found. These deaths, though presenting as SIDS, could no longer be certified as such as they did not completely match the definition. Causes of death in this group were infections (10 cases), Reye's syndrome (five), congenital cardiac defects (four), myocarditis (two) and one each of Waterhouse-Friderichsen syndrome, malnutrition, hypernatraemic dehydration, laryngeal cysts, and a ribbon impacted in the larynx.

The inclusion of cases into group $\mathrm{AB}$ relied on the judgment of the pathologist about the severity of the disease process present and the extent of its contribution to death. This was generally assessed histologically. As stated in one report, "there can be doubt and ambiguity, even after a thorough paediatric post-mortem, as to what constitutes an adequate cause of death'. ${ }^{6}$ Most postmortem examinations in this study were undertaken by the same prosector, which gave uniformity to both the performance and interpretation of the examination and contributed to the reliability of the findings. A pathogen was isolated and histological evidence of infection shown in 39 cases $(73.6 \%)$ in this group. In the other 14 cases $(26.4 \%)$ a variety of other diagnoses were found, such as a degree of nephrocalcinosis, inhalation of vomit, or metabolic disorders, but in no instance was the condition thought to be severe enough to be an adequate explanation for cause of death.

In most cases in group B there was a positive bacteriological or virological isolation but with no histological evidence of even minor infection. The classification B was used to show the presence of some positive finding, thus distinguishing the group from group $\mathrm{C}$, which had no positive finding.

\section{Results}

General factors. The greatest difference between any two groups in the proportion of homes that had no visit was found between groups $A$ and $A B$, but the difference was not significant $(p=0 \cdot 197$, Fisher's test of exact probability).

The social status of the family was determined by the occupation of the father, using the Office of Population Censuses and Surveys' Classification of Occupations. ${ }^{7}$ The only significant result obtained was that produced by a $\chi^{2}$ test applied to groups $\mathrm{B}$ and $C\left(\chi^{2}=7 \cdot 29, d f=2, p<0 \cdot 05\right)$. The most pronounced differences were in social classes one and two $(19 \%$ of group B and $34 \%$ of group C) and in social class three $(44 \%$ of group B and $34 \%$ of group C).

The family doctor, health visitor, and interviewer made an independent assessment of each family as regards parental supervision and management of the infant's feeding. The state of repair of the house and furnishings were also evaluated. Analysis of the gradings (good, average, or poor) produced a nonsignificant value for $F$ of $1.57(\mathrm{df}=3$ and 342$)$. Almost all estimates $(98 \%)$ were average or good.

Maternal factors. Analysis of the mothers' ages at the birth of the infants showed no significant differences between groups $(\mathrm{F}=2.90, \mathrm{df}=3$ and 353). There were also no significant differences between groups when mothers aged under 20 were compared $\left(\chi^{2}=2 \cdot 00, d f=3\right)$, the percentage of these young mothers in the groups ranging from $9 \%$ in group $\mathrm{AB}$ to $19 \%$ in group $\mathrm{A}$.

Over half $(53 \%)$ of the mothers smoked. The highest percentage of smokers was in group AB $(58 \%)$ and the lowest in group $\mathrm{C}(50 \%)$, but the differences were not significant $\left(\chi^{2}=0 \cdot 79, \mathrm{df}=3\right)$. 
Comparison of small for gestational age births ${ }^{8}$ in each group among smoking and non-smoking mothers produced a non-significant result $\left(\chi^{2}=5 \cdot 22\right.$, $\mathrm{df}=3$ ). Similarly, no significant difference was found when the infant was both small for gestational age and below the 10th centile at death $\left(\chi^{2}=0 \cdot 10, \mathrm{df}=1\right)$.

After the first born infants had been omitted the number born more or less than 16 months since the mother's last live birth were compared between groups. The only significant result was that $37 \%$ of group $\mathrm{A}$ had an interval of less than 16 months compared with $14 \%$ of group $B\left(\chi^{2}=5 \cdot 77, d f=1\right.$, $\mathrm{p}<0 \cdot 05$ ).

Most mothers (76.3\%) had booked with their own doctor or at the maternity hospital by 12 weeks' gestation, with the highest percentage not booking by this time in group A (33\%) and the lowest in group $\mathrm{C}(18 \%)$. The differences in booking times were not significant $\left(\chi^{2}=3 \cdot 976, \mathrm{df}=3\right)$.

Differences between the groups were small and non-significant $\left(\chi^{2}=0 \cdot 32, \quad \mathrm{df}=3\right)$ when antenatal complications were analysed, complications being present in $51 \%$ of group $\mathrm{AB}, 48 \%$ of group $\mathrm{C}, 48 \%$ of group $\mathrm{B}$, and $46 \%$ of group $\mathrm{A}$.

Infant factors. Group A contained the highest percentage $(38 \%)$ of first born infants compared with $19 \%$ in group $\mathrm{AB}$ and $27 \%$ in group $\mathrm{C}$. Proportions of second born infants were similar in groups $\mathrm{AB}, \mathrm{B}$, and $\mathrm{C}(57,46$, and $44 \%$, respectively), with the lowest representation in $A(31 \%)$. The highest representation of boys was in group $\mathrm{C}$ $(66 \%)$ and the lowest in group A $(50 \%)$, but these differences were not significant $\left(\chi^{2}=2 \cdot 56, d f=3\right)$.

The mean duration of gestation for the four groups ranged from 38.3 weeks (group A) to 38.9 weeks (group B), a non-significant difference by analysis of variance $(\mathrm{F}=1 \cdot 39, \mathrm{df}=3$ and 354$)$.

The mean birth weights ranged from $3154 \mathrm{~g}$ (group B) to $3052 \mathrm{~g}$ (group AB), but the difference was not significant by analysis of variance $(F=0 \cdot 32$, $\mathrm{df}=3$ and 349 ). Birth weights $\leqslant 1500 \mathrm{~g}$ represented from $1 \cdot 1 \%$ of group B to $3 \cdot 8 \%$ of group A.

The largest number of small for gestational age infants was in group $\mathrm{AB}(23 \%)$ and the smallest in group C $(13 \%)$. The difference was not significant $\left(\chi^{2}=3 \cdot 64, \mathrm{df}=3\right)$.

Differences for mean (SD) crown to heel length were small and not significant (analysis of variance, $\mathrm{F}=0.34, \mathrm{df}=3$ and 288 ), the shortest being $49 \cdot 65$, $(3.43) \mathrm{cm}$ (group AB) and the longest 50.40 (3.43) cm (group A).

Infant's health. Thirty per cent of group AB, $16 \%$ of group B, $15 \%$ of group C, and $12 \%$ of group A were admitted to the special baby unit some time after birth. Differences were not significant $\left(\chi^{2}=7 \cdot 168\right.$, $\mathrm{df}=3)$. Fewer infants in groups $\mathrm{A}$ and $\mathrm{AB}(15 \%$ and $4 \%$, respectively) were reported as healthy than in groups $\mathrm{B}$ and $\mathrm{C}$ ( $21 \%$ in both groups), the differences reaching significance $\left(\chi^{2}=9 \cdot 17, \quad d f=3\right.$, $\mathrm{p}<0 \cdot 05$ ).

Infants in group A were admitted to hospital twice as much as infants in groups $\mathrm{B}$ and $\mathrm{C}$, but this was not significant $\left(\chi^{2}=6 \cdot 43, \mathrm{df}=3\right)$.

When groups $\mathrm{A}$ and $\mathrm{AB}$ were compared with groups $B$ and $C$ about the health of the infants 48 hours before death significant differences were obtained. The percentage $(69 \%)$ of group A who were not healthy was significantly higher than group B $(40 \%)\left(\chi^{2}=8.03, \mathrm{df}=1, \mathrm{p}<0.01\right)$ and group $C$ $(30 \%) \quad\left(\chi^{2}=12 \cdot 73, \mathrm{df}=1, \mathrm{p}<0 \cdot 001\right)$. Similarly, the percentage of group $\mathrm{AB}$ who were not healthy $(60 \%)$ was significantly higher than group $\mathrm{B}$ $\left(\chi^{2}=7 \cdot 07, \mathrm{df}=1, \mathrm{p}<0.001\right)$ and group $\mathrm{C}\left(\chi^{2}=12.25\right.$, $\mathrm{df}=1, \mathrm{p}<0 \cdot 001)$.

Factors at death. Mean (SD) age at death was lowest in group $\mathrm{C}(16 \cdot 3(13 \cdot 2)$ weeks), highest in group $\mathrm{AB}$ (25.3 (20.9) weeks), and for group A was 22.0 $(20 \cdot 8)$ weeks and for group B was $18 \cdot 1,(114 \cdot 0)$ weeks). As there were significant differences between these means when analysed by an $F$ test $(F$ $4 \cdot 28, \mathrm{df}=3$ and $354, \mathrm{p}<0.05)$ a Scheffé test was employed to determine where these differences were. At death group $A B$ was significantly older than both group $B$ and group $C(p<0.05$ in both cases) and group A was significantly older than group B $(p<0 \cdot 01)$.

More infants died in the coldest months $(40 \%$ in December, January, and February). The number of deaths in these months within the groups ranged from $46 \%$ in group $\mathrm{A}$ to $34 \%$ in group C, but these differences were not significant $\left(\chi^{2}=1.99, \mathrm{df}=6\right)$.

The type of feeding at time of death showed a range for breast feeding alone from $31 \%$ (group A) to $13 \%$ (group $\mathrm{AB}$ ) and for bottle feeding alone from $62 \%$ (group B) to $49 \%$ (group C). Significantly more infants were bottle fed in group $B$ than in group $C\left(\chi^{2}=4 \cdot 01, d f=1, p<0 \cdot 05\right)$.

The mean interval from finding the infant dead to the time of postmortem examination for all cases was 38.9 hours. Infants below the 10 th centile on weight for age tables employed in hospitals where the postmortem examinations were performed comprised $52 \%$ of group $\mathrm{A}, 42 \%$ of group $\mathrm{AB}, 26 \%$ of group B, and $29 \%$ of group C. The difference between the groups was significant $\left(\chi^{2}=9 \cdot 93, d f=3\right.$, $\mathrm{p}<0.05$ ).

For the crown to heel measure, allowance was made for age by constructing $\mathrm{T}$ scores based on local 
norms for each age group. A non-significant result $(F=1 \cdot 56, d f=3$ and 272$)$ was obtained.

\section{Discussion}

Previous studies have shown that there are few, if any, differences between cases certified as sudden infant death syndrome and other cases reported to the coroner or procurator fiscal as unexpected deaths. ${ }^{9-13}$ Our study, which has found few significant differences between the groups based on degree of information, agrees with this.

Some features commonly associated with sudden infant death syndrome-namely, temporal variation, age distribution, and slight male predominance -were seen in our groups, but in contrast with most previous studies, there was no over representation of SIDS among families towards the lower end of the social class scale. The group as a whole was not marked by social deprivation, poor mothering, or less privileged families.

All infants in the study were reported as dying suddenly and unexpectedly. Classification took place only after postmortem examination and histological analysis. The present data indicated nonpathological differences between the groups, some of which may have been predicted, perhaps being an artefact of the criteria employed in classificationfor example, the pattern of incidence of reported ill health just before death in groups $A$ and $A B$.

Group A, because death was sudden and unexpected by history, could only match the adopted definition in that respect as a cause of death was found. For this reason they could not be classified as SIDS. This group produced the highest proportion of the main epidemiological factors associated with SIDS, thus supporting the finding that these variables are also associated with an increased incidence of all infant deaths. ${ }^{14}$

Most of the evidence for the influence of those variables commonly associated with increased risk of sudden infant death was found in groups $A$ and AB (Fig. 1). It has been well documented that children with conditions such as hypernatraemic dehydration and malnutrition are liable to come from homes where there is a considerable amount of substandard care, ${ }^{61516}$ but as there were only two such cases in our study it was considered that these did not introduce a selection bias.

These two groups ( $\mathrm{A}$ and $\mathrm{AB}$ ) also included a comparatively high representation of infants whose weight at postmortem examination fell below the 10th centile (Fig. 2). This may be partly due to postmortem dehydration, but as there was no longer or shorter death/necropsy interval between all the groups the presence of an underlying disease process may account for this finding. It has been suggested that growth patterns of some SIDS victims may be similar to those attributed to smoking during pregnancy.${ }^{17}$ Our data, which showed no significant difference between groups as regards maternal smoking, small for gestational age infants, and those who fell below the 10th centile, do not support this theory.

For purposes of certification of death in group $A B$ it may be more practicable to adopt the practice used in Sheffield, in which double registration enables a disease process to be identified and the death also to be described as SIDS. ${ }^{18}$ Like a previous study, ${ }^{19}$ we had reservations about including these subjects (group AB) in a 'true' SIDS group. Because of the element of doubt we decided, for the sake of the validity of the study, to form a separate group for these cases.

This group would match most closely 'Category B' as defined by Taylor and Emery, ${ }^{18}$ where factors

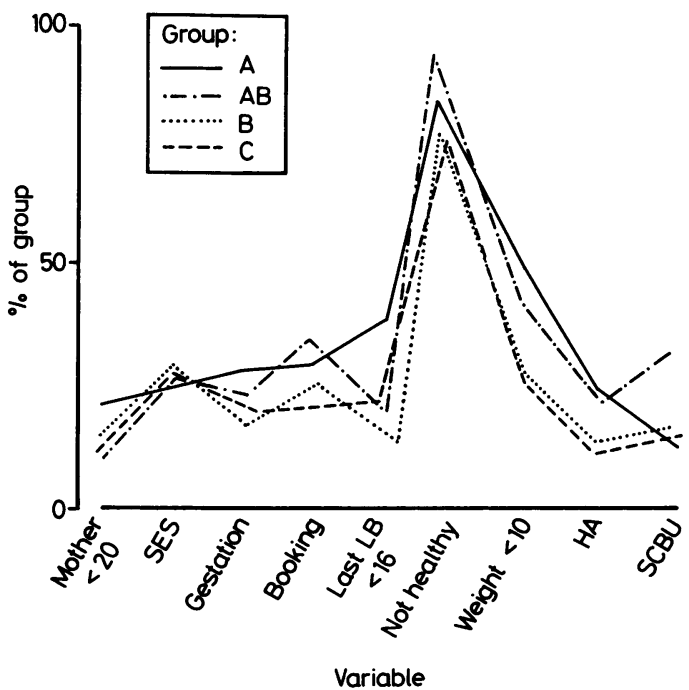

Fig. 1 Influence of variables commonly associated with increased risk of sudden infant death in the four groups (group $A=$ cases where a definite cause of death was ascertained $(n=26)$; group $A B=$ cases where an associated disorder was detected that may have been implicated $(n=53)$; group $B=$ cases where an associated disorder was detected but not implicated $(n=193)$; group $C=$ cases where no observable associated disorder was present $(n=86))$. Variables, reading left to right, are: Mother $<20$ years, socioeconomic state (SES), gestation $<37$ weeks (Gestation), booking time (at hospital) $<3$ months (Booking), last live birth $<16$ months ago (Last $L B<16$ ), infant not healthy, weight at postmortem examination $<10$ th centile (Weight $<10$ th), number of admissions to hospital $(H A)$, and admissions to special care baby unit (SCBU). Values are percentage of those in group with that variable. 
Small for gestational age

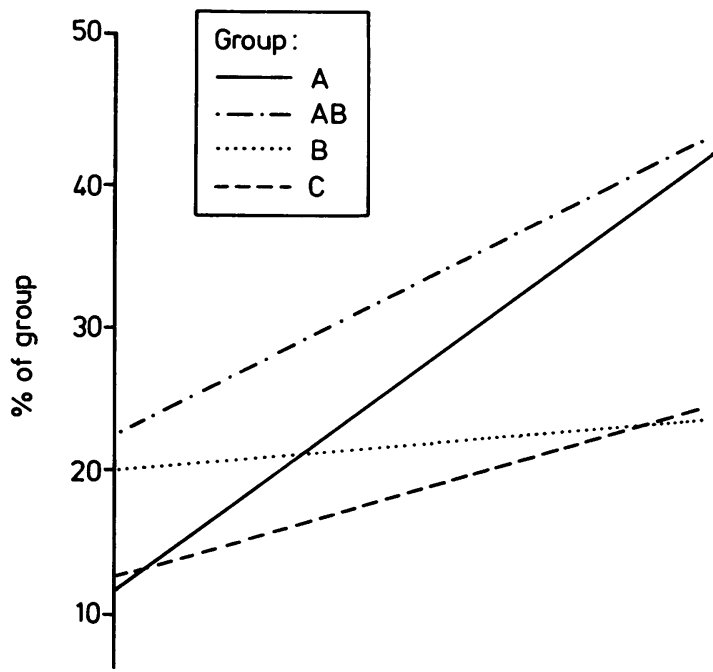

Fig. 2 Percentages of infants from the four groups (group $A=$ cases where a definite cause of death was ascertained $(n=26)$; group $A B=$ cases where an associated disorder was detected that may have been implicated $(n=53)$; group $B=$ cases where an associated disorder was detected but not implicated ( $n=193)$; group $C=$ cases where no observable associated disorder was present $(n=86))$ who were small for gestational age at birth and whose weight at postmortem examination fell below the 10th centile.

were found that were 'possibly treatable'. Death may have been preventable in some cases in group $\mathrm{AB}$ where it was possibly the result of an inappropriate response to infant illness by the caregiver or medical attendant. ${ }^{6}$ The inclusion and identification of a group 'AB' of this nature in SIDS studies may contribute to understanding the role of conventional paediatric treatment in the reduction of infant mortality.

The finding that there were less bottle fed infants in group $\mathrm{C}$ than in group $\mathrm{B}$ probably reflected social class, significantly more families from social classes I and II being in group C. These two groups were similar for most measures. Some factors commonly associated with SIDS, however, were apparent (second borns, age at death, and winter peaks) and in agreement with the findings in another study ${ }^{20}$ support for most attributed influences, such as high incidence of mothers aged less than 20 , short interpregnancy intervals, and premature birth, was not forthcoming. From these results it may be concluded that these 'true' SIDS infants would not appear as an 'at risk' population.
In Sheffield, where attempts have been made to identify infants at risk from unexpected death, the proportion of high and low risk infants varied considerably in different 'causal groups'. ${ }^{21}$ Compari-' son of this study with ours is difficult due to differences in classification, but there was a similar absence of 'risk' factors in the unexplained groups.

Compared with other infant deaths in Scotland, deaths due to SIDS are not significantly different when analysed by sex of child, social class of father, and age of mother. ${ }^{22}$ Our study supports Sunderland's view that all infants who die suddenly and unexpectedly are not always cases of SIDS. ${ }^{2.3}$ Many published reports on SIDS are confused by variation in definition. The term 'cot death' is often employed rather than SIDS but in fact only refers to the mode of death-that is, dying unexpectedly and suddenly. ${ }^{13}$

Bergman's definition ${ }^{1}$ was created to encompass those deaths that were sudden and unexpected and in which adequate cause had not been established. To date, we believe that this definition has not been bettered, even though it may be reconsidered as recommended in a recent report ${ }^{6}$ as it does not describe consistently the degree to which the death was unexplained.

The diagnosis of SIDS is one of exclusion of recognisable causes of death. Perhaps it should also include the consideration of adverse circumstances surrounding the death in the manner described by Bass et al, where fuller investigations into the circumstances surrounding death in 24 cases of certified SIDS changed the diagnosis in all instances. ${ }^{24}$ In the present study the circumstances surrounding death were fully investigated, by the police, before postmortem examination, and it is considered that the classification employed has permitted a group of 'true' SIDS to be identified (groups B and C), a group that also rigorously matched the standard definition of the syndrome set out above. The importance of a postmortem examination with prior and subsequent investigations provides a more reliable estimate of the incidence of SIDS. In areas where diagnosis is only made clinically - that is, where there is no autopsy-there may be over reporting or under reporting of the syndrome. An accurate diagnosis is beneficial in counselling bereaved parents and in the management of families who have or wish to have subsequent children, particularly about the question of the recurrence risk for SIDS and the use of apnoea monitors.

The diagnosis of SIDS may too often provide an 'umbrella' to cover cases where although death may have been sudden and unexpected, it was not always totally unexplained. 
In studies of sudden infant death there is often concern about the question of infanticide. The Infanticide Act does not apply in Scotland. Were there to be a charge it would almost certainly be that of culpable homicide. ${ }^{25}$ In the present study only two instances had some suspicion of parental mishandling.

'True' SIDS remains the largest single cause of death in infants who die suddenly and unexpectedly. Until a diagnostic marker or markers have been identified and agreed on, the diagnosis of SIDS remains dependent on the judgment of the pathologist. Misdiagnosis serves to confuse those seeking the cause(s) of these tragic deaths. This may give a falsely inflated picture of the incidence of the syndrome and also produce an unnecessarily high level of anxiety among parents.

\section{References}

1 Bergman AB, Beckwith JB, Ray GC, eds. Sudden infant death syndrome. Proceedings of 2nd international conference on causes of sudden death in infants. Scattle: University of Washington Press, 1970.

2 Kelly DH, Shannon DC. Sudden infant death syndrome and near sudden infant death syndrome: a review of the literature. 1964-1982. Pediatr Clin North Am 1982:29:5:1241-61.

3 Valdes-Dapena MA. Sudden infant death syndrome: a review of the literature, 1974-1979. Pediatrics 1980;66:597-614.

+ Freund JE. Mathematical statistics. New Jersey: Prentice Hall, 1962.

5 Scheffé H. The analysis of variance. New York: John Wiley, 1957.

"Knowelden J, Keeling J, Nicholl JP. A multicentre study of postneonatal mortality. London: DHSS, 1964.

7 Office of Population Censuses and Surveys. Classification of occupations. London: HMSO, 1970.

* Forfar JO. Arneil GC, eds. Textbook of Paediatrics. 3rd ed. Edinburgh: Churchill and Livingston, 1984:160.

" Richards IDG, McIntosh HT. Confidential inquiry into 226 consecutive infant deaths. Arch Dis Child 1972:47:697-706.
11) Cameron JM, Watson E. Sudden death in infancy in inner north London. J Pathol 1975;116:55-61.

1 McWeeny PM. Emery JL. Unexpected postnatal deaths (cot deaths) due to recognizable disease. Arch Dis Child 1975:50:191-6.

12 Froggatt P. Lynas MA. MacKenzic G. Epidemiology of sudden unexpected death in infants (cot death) in Northern Ireland. British Journal of Preventive and Social Medicine 1971;25: 119-34.

13 Arneil GC, Gibson AAM, McIntosh HT, Brooke H, Harvic A, Patrick WJA. National post-perinatal infant mortality and cot death study, Scotland 1981-82. Lancet 1985;i;740-3.

14 Moore A. The sudden infant death syndrome. Br J Hosp Med 1981;30:37-40.

15 Watson E, Gardner A. Carpenter RG. An epidemiological and sociological study of unexpected death in infancy in nine areas of southern England. 1: Epidemiology. Med Sci Law $1981 ; 21: 78-88$

16 Moore A. Preventable childhood deaths in Wolverhampton. $\mathrm{Br}$ Med J 1986;293:656-8.

17 Peterson DR. The sudden infant death syndrome-reassessment of growth retardation in relation to maternal smoking and the hypoxia hypothesis. Am J Epidemiol 1981;113:583-9.

is Taylor EM, Emery JL. Two year study of the causes of postperinatal deaths classified in terms of preventability. Arch Dis Child 1982;57:668-73.

19 Arncil GC, Gibson AAM, McIntosh HT, Brooke H, Harvie A, Patrick WJA. Post-perinatal infant mortality in Glasgow 1979 81. Lancet 1982;ii:649-51.

20) Radic A. Griffin M. Cahalane SF. Post neonatal mortality in Dublin with special considerations of sudden infant death syndrome. Ir Med J 1983;76:341-8.

2 Taylor EM, Emery JLE, Carpenter RG. Identification of children at risk of unexpected death. Lancet 1983;ii:1033-4.

22 Registrar General for Scotland. Deaths from sudden infant death syndrome, Scotland, 1979-83. London: HMSO, 1985.

${ }^{23}$ Sunderland R. Deaths in cots are not always cot deaths. Arch Dis Child 1985;60:156-72.

24 Bass M, Kravath MD. Glass MD. Death-scene investigation in sudden infant death. $N$ Engl J Med 1986:315:100-5.

25 Bain AD, Bartholomew, SEM. Sudden infant death syndrome in south-east Scotland. Lancet 1984;ii:1402.

Correspondence to S E M Bartholomew, Department of Pathology, Royal Hospital for Sick Children, Edinburgh EH9 1LF, Scotland.

Received 7 January 1987 